\title{
A brief assessment unravels unmet needs of older people in primary care: a mixed-methods evaluation of the SPICE tool in Portugal*
}

\author{
Conceição Balsinhaa ${ }^{1,2}$, Maria J. Marques ${ }^{2}$ and Manuel Gonçalves-Pereira ${ }^{2}$ \\ ${ }^{1}$ USF Marginal, São João do Estoril, Portugal \\ ${ }^{2}$ CEDOC, Chronic Diseases Research Centre, NOVA Medical SchoollFaculdade de Ciências Médicas, Universidade Nova \\ de Lisboa, Lisboa, Portugal
}

\begin{abstract}
Assessments of need may contribute to identifying health problems associated with functional deterioration in older people. A shorter version of the Camberwell Assessment of Need for the Elderly was developed for routine use in primary care, focusing on five domains: Senses, Physical ability, Incontinence, Cognition, and Emotional distress (SPICE). We aimed to explore its usefulness and feasibility in primary care.

We selected a consecutive sample of 51 community-dwelling older adults. The SPICE interview was completed by GPs and patients, with perceptions about its use in primary care being explored.

Needs were identified in 38 patients. Unmet needs corresponded to $7 \%$ of needs overall. 'Emotional distress' was the most frequent unmet need. SPICE helped to identify undisclosed needs, was well accepted and its importance in clinical evaluation recognised by GPs and patients, despite concerns about time constraints. Facilitating strategies are needed to improve the feasibility of these assessments in primary care.
\end{abstract}

Key words: primary care; needs assessment; ageing

Received 26 July 2017; revised 12 December 2017; accepted 20 December 2017;

first published online 21 January 2018

\section{Background}

The clinical evaluation of older people in primary care remains a challenging field. Comprehensive assessments combined with action on agreed problems can improve survival and function (Reuben et al., 1999; Harari et al., 2008; Ward and Reuben, 2012) but we still lack feasible approaches in general practice (Freer, 1985).

\footnotetext{
Correspondence to: Conceição Balsinha, Nova Medical School Faculdade de Ciências Médicas, Universidade Nova de Lisboa, Campo Mártires da Pátria 130, 1169-056 Lisboa, Portugal. Email: mbalsinha@gmail.com

* Partial results were presented as a poster ('Needs for care assessment is feasible: A study of elderly users in a Portuguese primary care setting') at the International Psychogeriatric Association Regional Meeting, December 2014, Brussels.
}

(Semi)structured assessments of need may identify health problems leading to functional deterioration. This patient-centred methodology allows the definition of health priorities and suitable interventions to address them (Wright et al., 1998). Unmet needs have been associated with low quality of life which calls for proactive needs assessments in clinical practice (Slade et al., 2005).

The Camberwell Assessment of Need for Elderly (CANE) (Reynolds et al., 2000; Orrell and Hancock, 2004) has been validated in different countries and settings (Walters et al., 2000; Ybarzabal Mesa et al., 2002; Kaiser et al., 2005). Being perhaps too lengthy for routine clinical use in primary care, a shorter version with five priority domains was developed (SPICE): Senses, Physical ability, Incontinence, Cognition and Emotional distress (Iliffe et al., 2004). 
Assessing needs in primary care entails a change in usual clinical practice. To our best knowledge, the use of brief needs assessments such as SPICE have never been tested in routine consultations. Our study aimed to explore the usefulness and feasibility of the SPICE assessment tool taking into account the perspectives of both GPs and patients.

\section{Methods}

\section{Design and participants}

This cross-sectional study was conducted in 2014, in one family health unit near Lisbon, with 11 GPs and 10 nurses responsible for 17800 registered patients (3692 aged $65+$ years). GPs approached all patients aged $65+$ years who attended the first scheduled appointment of the day, for six consecutive days. Patients on emergency visits were excluded, resulting in a sample of 51 patients.

\section{Needs assessment}

The SPICE interview was used, assessing: senses (vision and hearing), physical ability (mobility and falls), incontinence, cognition and emotional distress (Iliffe et al., 2004). These five items were extracted from the Portuguese translation of the CANE (Gonçalves-Pereira et al., 2007). In Portugal, the CANE was validated in a sample of older people with neuropsychiatric disorders (Fernandes et al., 2008) and used to interview nursing home residents (Ferreira et al., 2016), people with dementia (Kerpershoek et al., 2017) and people with psychiatric disorders (Passos et al., 2017).

According to the CANE instruction manual (Orrell and Hancock, 2004) and the literature (Reynolds et al., 2000; Walters et al., 2000), a trained rater provides a separate judgement for the patient and their carer, along with key-staff views on each domain and a fourth overall assessment (the rater's view). However, in order to study this assessment's feasibility in routine consultations, the obvious rater would be the GP, accumulating the role of 'key-staff'. To further document the usefulness of a proactive assessment in practice, we explored discrepancies between GPs' assumptions about users' needs (as based on previous consultations) and GPs' direct needs assessments in current consultations. Thus, in the present study, GPs provided three separate scores: (1) immediately before the consultation (GP-T0), using all available information in medical records; (2) scoring the patient's perspective during the consultation; and finally, (3) reporting again their own perspective after having interviewed the patients (GP-T1).

A 'met' need was recorded when a problem was identified and there was sufficient support to meet the need. An 'unmet' need was recorded when a problem was identified but there was either no support or insufficient support to meet the need (Orrell and Hancock, 2004). We did not consider interviewing the carers systematically because most patients would not have an informal carer, and the advantage of systematically doing this in clinical practice is still unknown (Walters et al., 2000).

All GPs were trained in the administration of SPICE, including through rehearsal in role-play scenarios. Pilot interviews were video-recorded and reliability training conducted.

\section{Additional feasibility data collection}

To address feasibility issues at different levels, we adopted a sequential explanatory mixed-methods design (Creswell, 2014) and complemented quantitative data with qualitative explorations of GPs' and patients' perceptions about the use of SPICE.

GPs' perceptions were assessed with a self-report questionnaire, including four multiple-choice items (on GPs' reactions, patients' reactions, perceived usefulness, and intentions regarding future use of the questionnaire) and two open-ended questions (further exploring advantages and difficulties).

Patients' perceptions were explored in individual interviews. While all the Unit's GPs were interviewed, we decided to randomise only five patients $(10 \%$ of the sample) due to operational constraints. These assessments took place at home or at the primary care facility as convenient, one week after each SPICE interview.

\section{Ethical issues}

Approval was obtained from ARS Lisboa e Vale do Tejo ethics committee. Participants and GPs gave their written informed consent.

\section{Data analysis}

Quantitative analysis was performed using IBM SPSS Statistics 21 (Mac Version). Cronbach's $\alpha$ was used to analyse SPICE internal consistency and $K$ tests to analyse levels of agreement between 
patients' and GPs' assessments of need. The significance level of $\alpha=5 \%$ was considered.

In the questionnaire for GPs, responses to multiple-choice items were analysed using absolute frequencies. Open-ended questions were subjected to content analysis, identifying ranges of responses organised according to predefined themes (advantages and difficulties). Additional thematic units derived from content analysis (11 sub-categories and 12 indicators).

The qualitative interviews with patients were conducted semi-structuredly, audio-taped and transcribed. Content analysis using a directed approach (Hsieh and Shannon, 2005) was employed to code the records into four predetermined categories (opportunity, advantages, difficulties, acceptability) or into new categories. Two researchers independently analysed and coded the transcripts, to improve data trustworthiness.

\section{Results}

SPICE interviews were completed for all eligible patients $(n=51)$. Demographic characteristics of the sample are illustrated in Table 1.

Table 1 Demographic characteristics of the sample $(n=51)$

\begin{tabular}{lr}
\hline Age (years) & \\
Mean (DP) & $74.9(7.3)$ \\
Age groups $n(\%)]$ & $25(49 \%)$ \\
65-74 years & $21(41 \%)$ \\
$75-84$ years & $5(10 \%)$ \\
85 or more years & \\
Education level $n(\%)]$ & $37(72 \%)$ \\
Low education & $7(14 \%)$ \\
Medium education & $7(14 \%)$ \\
High education & \\
Gender $[n(\%)]$ & $31(61 \%)$ \\
Female & $20(39 \%)$ \\
Male & \\
Civil status [ $n(\%)]$ & $1(2 \%)$ \\
Single & $27(53 \%)$ \\
Married & $4(8 \%)$ \\
Divorced & $19(37 \%)$ \\
Widowed & \\
Living arrangements $[n(\%)]$ & $15(29 \%)$ \\
Lives alone & $28(55 \%)$ \\
Lives with a spouse & $8(16 \%)$ \\
Lives with relatives & \\
Have a carer? $[n(\%)]$ & $6(12 \%)$ \\
Yes & $45(88 \%)$ \\
No & \\
\hline
\end{tabular}

Patients participating in individual interviews $(n=5)$ had a median age of 79.5 (range 66-88), and four were women. Two asked for the presence of a companion during the interview.

\section{Needs assessment: SPICE results}

All patients completed SPICE assessments. Median time taken for interviews was 8 min (range 3-23). In 11 (22\%) appointments there were other people present at the interview (two carers, nine relatives with no caring role).

Needs were identified by patients and their GPs (Table 2). Needs for care were reported by 38 (75\%) patients, reporting on average 1.6 needs each, 0.3 of which were, on average, unmet. Unmet needs $(n=17)$ corresponded to $7 \%$ of total needs $(n=255)$. 'Emotional distress' was the most frequent unmet need $(n=5)$.

Of 17 unmet needs identified by patients, six were unknown to GPs before consultations using SPICE.

According to Fleiss' (1981) criteria, $k$ tests between patients' and GPs'-T0 assessments showed fair agreement regarding senses $(k=0.59)$, incontinence $(k=0.56)$ and cognition $(k=0.58)$; and good agreement in physical ability $(k=0.68)$ and emotional distress $(k=0.71)$. $k$ tests between patients' and GPs'-T1 assessments showed excellent agreement in all domains (senses, $k=0.79$; physical ability, $k=0.79$; incontinence, $k=0.87$; cognition, $k=0.95$; emotional distress, $k=0.88$ ).

\section{GPs' and patients' perceptions about the use of SPICE}

The majority of GPs $(n=9)$ found that the information obtained from SPICE was quite useful or very useful, but only three intended to continue using SPICE in routine consultations (Table 3 ).

Regarding qualitative analysis, 'gaining knowledge' stood out of the five sub-categories of 'advantages' (Table 4).

Citing a GP, '... my views don't always correspond to the reality of older people'. SPICE allowed GPs to address key issues concerning older people's health and well-being: 'It allows preventive approaches towards wellbeing issues, usually forgotten by doctors'. Using SPICE also prompted inquiries about sensitive issues related with intimacy.

Concerning 'difficulties', six sub-categories emerged (Table 5). Time spent in assessments 
Table 2 Frequencies of needs identified using the SPICE by patients and general practitioners

\begin{tabular}{|c|c|c|c|c|c|c|}
\hline & \multicolumn{3}{|l|}{ Met needs } & \multicolumn{3}{|l|}{ Unmet needs } \\
\hline & Patients $(n=51)$ & GP-T0 ${ }^{\mathrm{a}}(n=51)$ & $\mathrm{GP}^{-\mathrm{T}} 1^{\mathrm{b}}(n=51)$ & Patients $(n=51)$ & $\mathrm{GP}^{-\mathrm{TO}^{\mathrm{a}}}(n=51)$ & $\mathrm{GP}^{-T} 1^{\mathrm{b}}(n=51)$ \\
\hline Senses & $24(47 \%)$ & $23(45 \%)$ & $24(47 \%)$ & $4(8 \%)$ & $4(8 \%)$ & $4(8 \%)$ \\
\hline Physical ability & $12(24 \%)$ & $10(20 \%)$ & $10(20 \%)$ & $1(2 \%)$ & $1(2 \%)$ & $2(4 \%)$ \\
\hline Incontinence & $6(12 \%)$ & $4(8 \%)$ & $5(10 \%)$ & $4(8 \%)$ & $3(6 \%)$ & $3(6 \%)$ \\
\hline Cognition & $12(24 \%)$ & $14(28 \%)$ & $13(26 \%)$ & $3(6 \%)$ & $0(0 \%)$ & $2(4 \%)$ \\
\hline Emotional distress & $12(24 \%)$ & $13(26 \%)$ & $13(26 \%)$ & $5(10 \%)$ & $2(4 \%)$ & $5(10 \%)$ \\
\hline
\end{tabular}

${ }^{\text {a }} \mathrm{GP}$ assessment before consultation.

${ }^{\mathrm{b}} \mathrm{GP}$ assessment after consultation.

Table 3 General practitioners' questionnaire multiple-choice questions results $(n=11)$

How do you describe your reaction to using SPICE?

$\begin{array}{lcccc}\text { Very bad } & \text { Bad } & \text { Reasonable } & \text { Good } & \text { Very good } \\ 0 & 0 & 3 & 4 \\ \text { How do you describe your patients' reaction to using } & \text { SPICE? } & \text { Good } & \text { Very good } \\ \text { Very bad } & \text { Bad } & 0 & \text { Reasonable } & 1\end{array}$

To what extent has the information obtained from SPICE been useful for the improvement of health care to your patients? Not at all useful Not very useful Somewhat useful Quite useful Very useful 0

$2 \quad 7 \quad 2$

How often will you continue to use SPICE on routine consultation with older patients?
Never
Seldom
Sometimes
6
Very often
3
Always
0

Table 4 Advantages identified by general practitioners on the use of the SPICE interview $(n=11)$

\begin{tabular}{|c|c|}
\hline Sub-categories & Indicators \\
\hline Gain knowledge of patients $(n=10)$ & $\begin{array}{l}\text { Doctor learns about their patient (eg, the support the person is } \\
\text { already receiving; ideas; worries and beliefs; needs) } \\
\text { Raise patients' awareness of their own health issues } \\
\text { Doctor learns about heterogeneity of older people } \\
\text { Giving voice to the patient }\end{array}$ \\
\hline $\begin{array}{l}\text { Address key issues concerning health and well- } \\
\text { being of the elderly }(n=8)\end{array}$ & $\begin{array}{l}\text { Forgotten issues } \\
\text { Undervalued issue }\end{array}$ \\
\hline $\begin{array}{l}\text { Promote a health/functionality centred approach } \\
(n=3)\end{array}$ & \\
\hline $\begin{array}{l}\text { Address potentially sensitive issues in a structured } \\
\text { and well-accepted manner }(n=2)\end{array}$ & $\begin{array}{l}\text { Personal experience of ageing } \\
\text { Meaning of life } \\
\text { Sexuality }\end{array}$ \\
\hline Improve care $(n=2)$ & \\
\hline
\end{tabular}

was the major one. To overcome time constraints, two GPs suggested that SPICE assessments should be completed throughout consecutive consultations. Despite previous training, distinguishing 'met' from 'unmet' need was deemed difficult in a few situations.

Regarding individual patient interviews, content analysis of the 'advantages' category identified 
Table 5 Difficulties identified by general practitioners on the use of SPICE interview $(n=11)$

\begin{tabular}{|c|c|}
\hline Sub-categories & Indicators \\
\hline \multicolumn{2}{|c|}{$\begin{array}{l}\text { Time spent in SPICE assessment given the duration of } \\
\text { consultation }(n=11)\end{array}$} \\
\hline Scoring SPICE interview $(n=7)$ & $\begin{array}{l}\text { Unable to discriminate scores of eyesight, hearing and } \\
\text { communication in the item 'senses' } \\
\text { Underlying concepts } \\
\text { Lack of training }\end{array}$ \\
\hline \multicolumn{2}{|c|}{ Assess the five areas in a single consultation $(n=3)$} \\
\hline \multicolumn{2}{|c|}{ Understand patients' responses $(n=2)$} \\
\hline \multicolumn{2}{|c|}{ Separately interviewing carer and patient $(n=1)$} \\
\hline $\begin{array}{l}\text { Absence of medical responses } \\
\text { presented }(n=1)\end{array}$ & \\
\hline
\end{tabular}

four sub-categories. Patients recognised that SPICE may improve the GP-patient relationship $(n=2)$, value issues important to patients $(n=2)$ and improve doctors' knowledge about patients $(n=2)$. Quoting a patient: 'I think that this consultation was clearly better, different from the others'. No patient reported discomfort about the issues addressed. In fact, all showed willingness to be questioned on these in future consultations and two even mentioned never having had previous opportunities to talk about some of them.

\section{Discussion}

\section{Main findings}

In this Portuguese primary care sample of older participants, SPICE disclosed information that was new to GPs. Furthermore, SPICE was well accepted and its importance recognised by GPs and patients alike.

To our knowledge, needs assessment of older patients in primary care had only been studied in the United Kingdom (Walters et al., 2000) and in Holland (Hoogendijk et al., 2014). Nevertheless, these studies differed from ours in important aspects: different versions of CANE used (24-item, Walters et al., 2000; 13-item, Hoogendijk et al., 2014); frail patients selected (Hoogendijk et al., 2014); interviews performed at home by trained interviewers, not by GPs themselves (Walters et al., 2000; Hoogendijk et al., 2014).

Exploring a routine scenario, GPs were the raters in our study and, despite small sample size, there were interesting findings. Better agreement in patient/GP post-assessment scores highlights the importance of GPs proactively asking about SPICE domains and not merely relying on their assumptions about their older patients. In fact, some unmet needs were unknown to GPs before SPICE assessments and this patientcentred approach helped to identify undisclosed needs, improving satisfaction in both patients and GPs.

\section{Feasibility}

All selected patients were interviewed $(n=51)$ and all SPICE items were completed $(n=255)$. The analysis of GPs' questionnaires showed that the majority of professionals accepted the SPICE interview well and valued the disclosed information. However, they were cautious regarding their intentions to use the interview in the future, probably because of the time needed to do so.

\section{Strengths and limitations}

Our study documented, for the first time, the usefulness and feasibility of a brief needs assessment designed for primary care but never actually tested. However, it was conducted in a single, local setting, with a small non-randomized sample, and a major limitation is lack of generalisability.

Using mixed methods allowed us to explore patients' and professionals' perceptions, expanding the analysis of the SPICE assessment's feasibility. However, pragmatic reasons limited the number of patient interviews, whose findings were mainly about SPICE use advantages. Further probing and additional interviews would have been needed to explore patients' views more fully. 
Although the CANE interview was validated in Portugal (Fernandes et al., 2008), this SPICE version was used for the first time in Portuguese primary care. Indeed, its psychometric properties should be further evaluated internationally as only the full CANE has been applied in primary care in other countries (Walters et al., 2000).

The triangulation of perspectives is an asset of CANE (Orrell and Hancock, 2004); however, some argue that its usefulness in clinical practice is not yet established (Walters et al., 2000). Although we have not interviewed carers in this study, it might have been important in some cases.

\section{Implications for clinical practice and policy}

SPICE can unravel unexpressed needs for care, although the extended length of consultations may be a source of concern. Its practical implementation should be further evaluated, probably requiring top-down and bottom-up strategies altogether. Being well accepted overall by physicians and patients, perhaps its use by primary care nurses could contribute to overcoming GPs' time constraints.

Although SPICE domains relate to older people's functionality (Stuck et al., 1999), we lack direct evidence that the routine use of this interview specifically builds effective strategies for improving clinical outcomes.

\section{Conclusions}

SPICE interviewing may yield new valuable information about unmet needs of older people in primary care, but its implementation may not be straightforward and requires facilitating strategies.

\section{Acknowledgements}

The authors thank all the doctors of USF Marginal: A. Ferrão, A. Franco, A. Biscaia, J. Faria, J. Ramires, J. Sousa, M. Santos, P. Charondière, T. Carneiro and V. Ramos. This study was presented by the first author as part of a Master's Thesis (NOVA Medical School/Faculdade de Ciências Médicas - Universidade Nova de Lisboa). J. Grave provided valuable help editing the manuscript.

\section{Financial Support}

None.

\section{Conflicts of Interest}

None.

\section{References}

Creswell, J.W. 2014: Mixed methods procedures. In Creswell J.W., editor Research design - quantitative, qualitative and mixed methods approaches, fourth edition. Los Angeles: SAGE Publications, Inc, 264-88.

Fernandes, L., Gonçalves-Pereira, M., Leuschner, A., Martins, S., Sobral, M., Azevedo, L.F., Dias, C., Mateos, R. and Orrell, M. 2008: Validation study of the Camberwell Assessment of Need for the Elderly (CANE) in Portugal. International Psychogeriatrics 21, 94-102.

Ferreira, A.R., Dias, C.C. and Fernandes, L. 2016: Needs in nursing homes and their relation with cognitive and functional decline, behavioral and psychological symptoms. Frontiers in Aging Neuroscience 8, 72, doi: 10.3389/ fnagi.2016.00072.

Fleiss, J. 1981: The measurement of inter-rater agreement. In Fleiss J., editor Statistical methods for rates and proportions. New York: John Wiley \& Sons, 42-52.

Freer, C.B. 1985: Geriatric screening: a reappraisal of preventive strategies in the care of the elderly. Journal of the Royal College of General Practitioners 35, 288-90.

Gonçalves-Pereira, M., Fernandes, L., Leuschner, A., Barreto, J., Falcão, D., Firmino, H., Mateos, R. and Orrell, M. 2007: Versão Portuguesa do CANE (Camberwell Assessment of Need for the Elderly): desenvolvimento e dados preliminares. Revista Portuguesa de Saúde Pública 5, 7-18.

Harari, D., Iliffe, S., Kharicha, K., Egger, M., Gilmann, G., von Renteln-Kruse, W., Beck, J., Swift, C. and Stuck, A. 2008: Promotion of health in older people: a randomised controlled trial of health risk appraisal in British general practice. Age and Ageing 37, 565-71.

Hoogendijk, E.O., Muntinga, M.E., van Leeuwen, K.M., van der Horst, H.E., Deeg, D.J.H., Frijters, D.H.M., Hermsen, L.A.H., Jansen, A.P.D., Nijpels, G. and van Hout, H.P.J. 2014: Self-perceived met and unmet care needs of frail older adults in primary care. Archives of Gerontology and Geriatrics 58, 37-42.

Hsieh, H.F. and Shannon, S.E. 2005: Three approaches to qualitative content analysis. Qualitative Health Research 15 , 1277-288.

Iliffe, S., Lenihan, P., Orrell, M., Walters, K., Drennan, V. and Tai, S.S., SPICE Research Team 2004: The development of a short instrument to identify common unmet needs in older people in general practice. British Journal of General Practice 54, 914-18. 
Kaiser, G., Krautgartner, M., Rainer, A., Unger, A., Marquart, B., Weiss, M. and Wancata, J. 2005: Die Ubereinstimmungsvalidaitat des 'Carers' Needs Assessment for Dementia' (CNA-D). Neuropsychiatrie 19, 134-40.

Kerpershoek, L., de Vugt, M., Wolfs, C., Woods, B., Jelley, H., Orrell, M., Stephan, A., Bieber, A., Meyer, G., Selbaek, G., Handels, R., Wimo, A., Hopper, L., Irving, K., Marques, M., Gonçalves-Pereira, M., Portolani, E., Zanetti, O., Verhey, F. and Actifcare Consortium 2017: Needs and quality of life of people with middle-stage dementia and their family carers from the European Actifcare study. When informal care alone may not suffice. Aging and Mental Health 1-6, doi: 10.1080/13607863.2017.1390732.

Orrell, M. and Hancock, G. 2004. The Camberwell Assessment of Need for the Elderly (CANE). London: Gaskell.

Passos, J., Fonte, A., Dias, C.C. and Fernandes, L. 2017: The needs of older people with mental health problems in a Portuguese psychiatric setting. International Psychogeriatrics 29, 845-53.

Reuben, D.B., Frank, J.C., Hirsch, S.H., McGuigan, K.A. and Marly, R.C. 1999: A randomized clinical trial of outpatients comprehensive geriatric assessment coupled with an intervention to increase adherence to recommendations. Journal of the American Geriatrics Society 47, 269-76.

Reynolds, T., Thornicroft, G., Abas, M., Woods, B., Hoe, J., Leese, M. and Orrell, M. 2000: Camberwell Assessment of
Need for the Elderly (CANE). Development, validity and reliability. The British Journal of Psychiatry 176, 444-52.

Slade, M., Leese, M., Cahill, S., Thornicroft, G. and Kuipers, E. 2005: Patient-rated mental health needs and quality of life improvement. The British Journal of Psychiatry 187, 256-61.

Stuck, A.E., Walthert, J.M., Nikolaus, T., Bula, C.J., Hohmann, C. and Beck, J.C. 1999: Risk factors for functional status decline in community-living elderly people: a systematic literature review. Social Science \& Medicine 48, 445-69.

Walters, K., Iliffe, S., Tai, S.S. and Orrell, M. 2000: Assessing needs from patient, carer and professional perspectives: the Camberwell Assessment of Need for Elderly people in primary care. Age and Ageing 29, 505-10.

Ward, K.T and Reuben, D.B. 2012: Comprehensive geriatric assessment. Retrieved 11 July 2013 from http://www. uptodate.com/contents/comprehensive-geriatric-assessment? source=search_result\&search=geriatric+assessment\&selected Title $=1 \% 7 E 46$.

Wright, J., Williams, R. and Wilkinson, J.R. 1998: Health needs assessment: development and importance of health needs assessment. British Medical Journal 316, 1310-313.

Ybarzabal Mesa, M., Mateos, R., Jesús Garcia, M., Amboage, M. and Fraguela Pérez, I. 2002: Validacion de la version espanola del CANE. Escala de Evaluacion de Necesidades para Ancianos de Camberwell. Revista de Psicogeriatria 2, 38-44. 